\title{
Composición y tamaño de la red de apoyo social en distintas etapas vitales
}

\author{
Composition and Size of the Social Support Metwork in Different Life Stages \\ Composição e dimensão da rede social de apoio nas diferentes fases da vida
}

\author{
Claudia J. Arias \\ Belén Sabatini \\ Mirta Scolni \\ Tamara Tauler \\ Universidad Nacional de Mar del Plata
}

Doi: https://doi.org/10.12804/revistas.urosario.edu.co/apl/a.7901

\section{Resumen}

La red de apoyo social es de gran importancia a lo largo de la vida, pues incide sobre el bienestar integral. Sin embargo, esta se modifica durante el curso vital y su tamaño tiende a disminuir en la vejez. El objetivo de este trabajo fue explorar su composición y tamaño en distintas etapas vitales: juventud (20 a 30 años), mediana edad (40 a 50 años), vejez (60 a 70 años) y vejez avanzada ( 80 a 90 años). Se implementó un diseño no experimental transversal correlacional. A una muestra no probabilística intencional de 400 sujetos residentes en Mar del Plata (100 de cada grupo de edad) se le administró un cuestionario de datos sociodemográficos y la Entrevista Estructurada de Fuentes de Apoyo Social (Vaux \& Harrison, 1985). Los datos fueron analizados mediante el programa R versión 3.4.1. Se utilizaron distribuciones de porcentajes para comparar la composición, así como la prueba $t$, el ANOVA y el contraste de Bonferroni para identificar diferencias entre los grupos. Los resultados muestran que las amistades, la pareja y los hermanos/as son los vínculos más presentes en todas las etapas vitales. El tamaño de la red de apoyo social es más elevado en los grupos de menos edad. Los promedios significativamente más altos de amigos/ as, de compañeros/as de trabajo/estudio y de relaciones comunitarias/servicios de salud correspondieron a los jóvenes, adultos de mediana edad y de vejez avanzada respectivamente. Se concluye que la red presenta cambios a lo largo del curso vital que podrían impactar en el bienestar integral.

Palabras clave: red de apoyo social; curso vital; evaluación; estructura; composición.

\section{flbstract}

The social support network is of great importance throughout life, influencing comprehensive well-being. However, it changes over life and tends to decrease in size in old

Claudia J. Arias ORCID ID: https://orcid.org/0000-0003-2154-187X

Belén Sabatini ORCID ID: https://orcid.org/0000-0002-0059-3139

Mirta Scolni ORCID ID: https://orcid.org/0000-0001-9923-9836

Tamara Tauler ORCID ID: https://orcid.org/0000-0001-7031-5727

Dirigir correspondencia a Claudia J. Arias. Correo electrónico: cjarias@mdp.edu.ar

Para citar este artículo: Arias, C. J., Sabatini, B., Scolni, M., \& Tauler, T. (2021). Composición y tamaño de la red de apoyo social en distintas etapas vitales. Avances en Psicología Latinoamericana, 38(1), 1-15. https://doi.org/10.12804/revistas.urosario.edu.co/apl/a.7901 
age. The aim of this work was to explore its composition and size in the different life stages: youth (20 to 30 years old), middle-age (40 to 50 years old), old age (60 to 70 years old), and advanced old age ( 80 to 90 years old). To do this, we introduced a crosscutting, non-experimental design. To an intentional non-probabilistic sample of 400 research subjects, residing in Mar del Plata (100 out of each age group), we applied a sociodemographic questionnaire and the Structured Interview of Sources of Social Support (Vaux \& Harrison, 1985). The data were analyzed through R version 3.4.1. Distributions of percentages to compare the composition as well as the t-test, the ANOVA, and Bonferroni's contrast were used to identify differences between groups. The results show that friendship, partners, and siblings are the most included bonds at all life stages. At less age, the size of the social support network is bigger than at other ages. The higher average of friends, co-workers/study, and community/health services relation belongs to young people, middle-aged, and old-aged adults, respectively. It is concluded that the network shows changes throughout life, which can affect well-being.

Keywords: Social support network; life course; evaluation; structure; composition.

\section{Resumo}

A rede de apoio social é de grande importância ao longo da vida, influenciando no bem-estar integral. No entanto, ela é modificada ao longo da vida e tende a diminuir de tamanho na velhice. O objetivo deste trabalho foi explorar sua composição e tamanho em diferentes fases da vida: juventude ( 20 a 30 anos), meia-idade (40 a 50 anos), velhice (60 a 70 anos) e velhice avançada (80 a 90 anos). Um desenho correlacional transversal não experimental foi implementado. A uma amostra não probabilística intencional de 400 indivíduos residentes em Mar del Plata (100 de cada faixa etária) foi aplicado um questionário de dados sociodemográficos e a Entrevista Estruturada de Fontes de Apoio Social (Vaux \& Harrison, 1985). Os dados foram analisados por meio do programa R versão 3.4.1. Distribuições percentuais foram utilizadas para comparar a composição, bem como teste t, ANOVA e o contraste de
Bonferroni para identificar diferenças entre os grupos. Os resultados mostram que amigos, parceiros e irmãos são os elos mais incluídos em todas as fases da vida. $\mathrm{O}$ tamanho da rede de apoio social é maior nas faixas etárias mais jovens. As médias significativamente mais altas para amigos, colegas de trabalho / estudo e de relações comunitárias / serviços de saúde corresponderam a jovens, adultos de meia-idade e idade avançada, respectivamente. Conclui-se que a rede apresenta mudanças ao longo do curso da vida que podem impactar no bem-estar integral.

Palabras clave: rede de apoio social; curso da vida; avaliação; estrutura; composição.

El apoyo social puede ser entendido como el conjunto de transacciones interpersonales que implican ayuda, afecto o afirmación (Kahn \& Antonucci, 1980). Desde los años 70, las investigaciones sobre el apoyo social generaron una abundante producción tanto a nivel teórico, como interventivo (Dabas, 1993; Gracia Fuster, 1997; Lomnitz, 1975). Asimismo, los organismos internacionales interesados en la vejez y el envejecimiento han destacado la importancia del fortalecimiento de los apoyos sociales debido a su impacto, notable y ampliamente documentado en la literatura científica, sobre la salud y el bienestar integral a lo largo del curso vital (CEPAL, 2004; Naciones Unidas, 2002; OMS, 2002).

El estudio e intervención desde el apoyo social sostiene una concepción de salud positiva; apunta al cambio social, teniendo en cuenta la participación activa de los sujetos involucrados en el problema; se interesa por el fortalecimiento y desarrollo de los individuos, los grupos y la comunidad; le otorga más importancia a las potencialidades, competencias y recursos que a los déficits, y posee un importante interés preventivo (Sánchez Vidal, 1990). Según Sluzki (1996), los apoyos sociales ayudan a "proveer sentido a la vida de sus miembros [...], [aportan] retroalimentación cotidiana acerca de las desviaciones de la salud [...] y propician una serie 
[de] actividades personales que se asocian positivamente con sobrevida" (p. 81). Diversos estudios han evidenciado su relación con el bienestar físico (Cohen, 2020; Ye \& Zhang, 2019) y psicológico en la vejez (Gyasi et al., 2019; Huang et al., 2019).

Entre los apoyos informales de los que dispone una persona se encuentra la red de apoyo social, la cual está conformada por un conjunto restringido de relaciones familiares y no familiares que brindan alguna o varias formas de ayuda. Se puede disponer de ella tanto de manera cotidiana como en situaciones críticas y resulta fundamentalmente útil para el desarrollo de soluciones creativas frente a conflictos y problemas. La red posee características estructurales - tamaño, distribución y densidad - así como funcionales - apoyo emocional, social, económico, práctico y de orientación/ guía-. Asimismo, los vínculos que la conforman presentan una serie de atributos - eg., intimidad, historia, frecuencia de contacto, multidimensionalidad - que pueden ser evaluados con variados instrumentos de exploración.

A su vez, la red puede ser mapeada mediante una representación gráfica que permite ubicar a los vínculos que la integran en 12 sectores que surgen de la intersección de los cuadrantes correspondientes a 1) relaciones familiares, 2) amistades, 3) relaciones comuntarias/servicios de salud y 4) relaciones laborales/de estudio con tres círculos concéntricos que diferencian niveles de intimidad elevados, medios y bajos (Sluzki, 1996).

En cuanto a las modificaciones que puede atravesar la red de apoyo en las distintas etapas vitales, Sluzki (1996) refiere que esta presenta numerosos cambios a lo largo del curso vital, que suponen variaciones en el apoyo del cual se dispone. Según el autor, las redes atraviesan una etapa de expansión durante la niñez y la adolescencia, de estabilización en la adultez y de contracción en la vejez.

Al respecto, diferentes teorías han tratado de explicar estos cambios. En ese sentido, la teoría de la selectividad socioemocional de Carstensen (2006) sostiene que las personas mayores tienden a restrin- gir sus redes a medida que crecen. La percepción de la finitud las motiva a concentrar sus energías afectivas en las personas que son más importantes para ellas. Las metas afectivas y emocionales tienden a ser cada vez más selectivas y se priorizan sobre otras, como la adquisición de información y la expansión de horizontes. Por lo tanto, desde dicha teoría, los adultos mayores asumen un rol proactivo en sus relaciones sociales (Carstensen, 2006). De este modo, la disminución de los miembros que conforman la red en la vejez es el resultado de una elección intencional de los adultos mayores que estratégicamente seleccionan los vínculos que más les interesa seguir manteniendo y profundizando. Ciertas pérdidas de vínculos pueden compensarse con ganancias de apoyo emocional y de contacto con los lazos que se vuelven más íntimos. Esto permite pensar que la red social se modifica no solo en tamaño, sino también en su composición y en los atributos de sus vínculos. Baltes y Freund (2007) proponen que frente a las pérdidas se desarrollan procesos de selección, optimización y compensación que permiten la adaptación a la nueva situación, así como el mantenimiento del nivel de bienestar anterior a ellas.

En esta misma línea de investigación, Kahn y Antonucci (1980) desarrollaron el modelo de convoy de las relaciones sociales. El término convoy fue acuñado por Plath (1980) para dar cuenta de la cohorte de vida y de aprendizaje en la que se criaban niños japoneses, pretendiendo transmitir la función protectora de las relaciones sociales al transitar ciertos acontecimientos a lo largo de su vida. Asimismo, Kahn y Antonucci (1980) usaron el término para dar cuenta del curso vital de las relaciones sociales. Antonucci (2001) sostiene que las personas desarrollan y abandonan una variedad de roles a lo largo de sus vidas y que cada uno de ellos está acompañado por un conjunto especifico de relaciones. Existen ciertos patrones que son consistentes con determinados eventos en el curso vital; por ejemplo, en la vejez, la jubilación y la pérdida por fallecimiento de la pareja o 
de otros vínculos podrían disminuir el tamaño de la red o el nacimiento de nietos provocar nuevas incorporaciones. Asimismo, en la mediana edad, la importancia de la crianza de los hijos y de la vida laboral impactará en la conformación de la red que incluirá muy probablemente a amigos y compañeros de trabajo, y también relaciones establecidas en ámbitos escolares o deportivos en los que sus hijos participan (Arias, 2019). Además, las relaciones varían en su cercanía, calidad, función - ayuda, afecto, intercambios de afirmación - y estructura - eg. tamaño, composición, frecuencia de contacto, proximidad geográfica - . La estructura, la función y la calidad de dicho convoy están influenciadas por características personales (eg., la edad y el género) y situacionales (las demandas de rol, las normas, los valores) al tiempo que tienen implicaciones significativas para la salud y el bienestar (Antonucci et al., 2014). Este convoy da cuenta de una red activa y dinámica, que varía con el tiempo y puede experimentar distintas modificaciones en cada etapa vital.

Los hallazgos acerca del dinamismo de la red social a lo largo del curso vital resultan diversos. Algunos muestran que en la vejez se producen numerosas pérdidas de vínculos, fundamentalmente por fallecimiento de amigos y de familiares, por enfermedades y por pérdida de contacto, que reducen notablemente el número de relaciones en la vejez avanzada (Conway et al., 2013). Otros destacan que, si bien se producen pérdidas, también se mantienen y optimizan relaciones que han sido establecidas en etapas anteriores de la vidas y se incorporan nuevos vínculos (Cornwell \& Laumann, 2013) dando cuenta de la coexistencia de pérdidas y de ganancias en la vejez (Arias, 2015a; 2015b), así como de la satisfacción con el apoyo del que disponen (Acrich, 2012).

Diversos estudios han puesto en evidencia que existe una amplia variabilidad en lo referido al tamaño y a la composición de las redes en los adultos mayores, y que gran parte de ellos dispone de recursos de apoyo variados y adecuados (Arias,
2004; 2009; Pantusa \& Arias, 2017; Cornwell et al., 2009; Fernandez Ballesteros, Reig Ferrer \& Zamarron, 2009). Al respecto, Carstensen y Charles (2007) sostienen que las explicaciones que se han brindado acerca de la disminución de las relaciones sociales en la vejez se han basado más en ideas y estereotipos —en muchos casos incorrectos - que en hallazgos de investigaciones.

En un estudio que exploró los aspectos estructurales y funcionales de las redes de apoyo social de personas mayores, realizado en Mar del Plata, Argentina, se encontró una gran heterogeneidad en las mismas, reafirmando la variabilidad interindividual del proceso de envejecimiento y desestimando el supuesto aislamiento social en la vejez como un hecho generalizado (Arias, 2013; 2015b).

En lo referido a la composición de las redes, recientes hallazgos han mostrado que entre los apoyos que recibe la persona mayor, los hijos/as, la pareja y los amigos/as han sido identificados no solo como los de inclusión más frecuente, sino también como los que brindan más satisfacción a las personas mayores (Arias, 2004; Polizzi \& Arias, 2014). Algunos estudios muestran que aquellos que viven con sus familias presentan mejores niveles de salud mental, física y emocional que aquellos que viven solos (Bozo et al., 2009; Vivaldi \& Barra, 2012). Otros sostienen que la ausencia de familiares cuando se dispone del apoyo de amigos es menos perjudicial que la ausencia de amigos cuando se dispone del apoyo de familiares (Fiori et al., 2006).

Dentro de los vínculos familiares, las investigaciones muestran que la pareja es un vínculo central en la vejez (Becker et al., 2019). Mientras los varones consideran que dicho vínculo les proporciona principalmente apoyo emocional, las mujeres destacan que les proveen de ayuda práctica y económica (Arias \& Polizzi, 2013). Asimismo, los primeros reciben más apoyo de sus parejas (Reyes et al., 2006), mientras que las mujeres tienden a priorizar a los hijos como suministradores de apoyo (Meléndez Moral et al., 2007). Respecto a la relación de amistad, las investigaciones enfati- 
zan la función socializadora que tiene este tipo de vínculo en la vejez (Arias, 2013) así como su asociación con la longevidad (Giles et al., 2005) y la reducción del riesgo de morir por cualquier causa (Shirom et al., 2011).

La investigación de las redes en otras etapas vitales es más escasa. En la juventud, su exploración se ha centrado en relacionar el apoyo social con la depresión (Okamoto et al., 2011), la autoestima (Lee et al., 2014), la ideación suicida (Gallagher et al., 2014) y las conductas sexuales de riesgo (Orcasita et al., 2012). Asimismo, se han explorado ampliamente las características de las redes de apoyo social en adolescentes que abandonan el hogar familiar (Johnson, Whitbeck \& Hoyt, 2005). De acuerdo con Frey y Roethlisberger (1996), las relaciones con pares proveen de apoyo en cuestiones cotidianas mientras que el apoyo de los padres y demás familiares posee un efecto amortiguador del estrés en situaciones de emergencia.

Los estudios sobre las redes de apoyo social en la mediana edad son aún más reducidos. Sin embargo, algunos casos que han incluido en su muestra a personas que transitan esta etapa vital no han hallado diferencias significativas en el tamaño de las redes, en comparación con las de personas adultas mayores. En una investigación realizada en Estados Unidos sobre una muestra representativa de adultos a nivel nacional que superaba los 3000 casos, distribuidos en tres grupos de edad ( 57 a 64 años, 65 a 74 años y 75 a 85 años), se comparó la cantidad de personas a quienes los entrevistados consideraban confidentes y con los que podían hablar sobre temas importantes. Las medias obtenidas fueron de 3.5 para el grupo más joven y de 3.4 para el de más edad, sin diferencias significativas entre ellos a pesar de existir un rango etario amplio entre ambos (Cornwell et al., 2009).

A partir de lo expuesto y teniendo en cuenta la escasez de antecedentes con respecto a las diferencias en la red de apoyo en diferentes etapas vitales, surge el interrogante acerca de cuál es su tamaño y composición en personas de cuatro grupos de edad. Se plantearon como objetivos: 1) Identificar los vínculos incluidos más frecuentemente (padre, madre, hijo/a, pareja, hermano,/a, amigos/as, etc.) en la red en cada grupo de edad; 2) Determinar si existen diferencias significativas en el tamaño de la red por grupo de edad; 3) Evaluar si existen diferencias significativas según grupo de edad en las puntuaciones medias de cantidad de: a) amistades, b) familiares, c) relaciones comunitarias y servicios de salud y d) compañeros/as de trabajo o estudio.

Los resultados permitieron identificar los aspectos en los que la red de apoyo social presenta diferencias y similitudes en distintas etapas de la vida, así como efectuar aportes para el diseño de intervenciones que promuevan el bienestar considerando los recursos y falencias existentes en cada una de ellas.

\section{Metodología}

\section{Diseño}

Se trabajó con un diseño no experimental de tipo transversal correlacional.

\section{Participantes}

La población en estudio estuvo constituida por personas de distintas etapas vitales: 1$)$ jóvenes (20 a 30 años), 2) adultos de mediana edad (40 a 50 años), 3) adultos mayores jóvenes (60 a 70 años) y 4) adultos mayores de edad avanzada (80 a 90 años), de ambos sexos que vivían de manera permanente en la ciudad de Mar del Plata y no presentaban deterioro cognitivo aparente. Fueron excluidos de la muestra aquellos casos en que los evaluadores observaron la presencia de indicadores de dicha patología. Para la descripción de la muestra se indagó la edad, la ocupación, el grupo de convivencia y el nivel educativo (tabla 1 ).

Los grupos de edad fueron considerados con la finalidad de incluir diferentes etapas vitales y con una distancia de edad entre ellos que permitiera 
apreciar las diferencias si las hubiere. Se seleccionó una muestra no probabilística intencional conformada por 400 sujetos -100 de cada grupo de edad (50 varones y 50 mujeres) — . El tamaño de la muestra y la cantidad de casos en cada grupo fueron determinados de acuerdo con los criterios para testear hipótesis sustantivas (Padua, 1982). De este modo, se garantiza un número adecuado de unidades de análisis para la aplicación de las técnicas estadísticas requeridas.

\section{Instrumentos}

En el trabajo de campo se utilizaron las siguientes técnicas para la recolección de datos:

Cuestionario de datos sociodemográficos: mediante preguntas cerradas se exploraron variables de base que permitieron caracterizar la muestra seleccionada: género, edad, conformación del grupo de convivencia, nivel educativo y ocupación.

\section{La Entrevista Estructurada de Fuentes de} Apoyo Social (Vaux \& Harrison, 1985): esta evalúa variados aspectos de la red. Se pide al entrevistado/a que nombre a las personas que le brindan los distintos tipos de apoyo describiéndoles situaciones típicas a modo de ejemplificaciones que faciliten la identificación de las relaciones en cuestión. Una vez detectadas dichas personas, se elabora el listado total de relaciones que posee y se completa una serie de datos de cada una de ellas, que permiten identificar específicamente diversos atributos de cada vínculo. Entre las características de la red que se pueden evaluar se encuentran el tamaño, definido como la cantidad total de personas incluidas como parte de la red - es decir, que brindan alguna función de apoyo- e identificar los miembros que la conforman por tipo de relación (hijo/a, amigo/a, pareja, nieto/a, hermano/a, primo/a, vecino/a, compañero/a, etc.). A su vez, esta información posibilita conocer el número de amistades, familiares,

Tabla 1.

Características sociodemográficas de los participantes por grupo de edad

\begin{tabular}{|c|c|c|c|c|}
\hline Características & 20 a 30 años n: 100 & 40 a 50 años n: 100 & 60 a 70 años n: 100 & 80 a 90 años n: 100 \\
\hline \multirow{2}{*}{ Edad } & $\mathrm{M}: 25$ & M: 45 & M: 64 & M: 83 \\
\hline & DS: 3.72 & DS: 3.34 & DS: 3.47 & DS: 2.45 \\
\hline \multicolumn{5}{|l|}{ Grupo conviviente } \\
\hline Solo & 13 & 23 & 29 & 39 \\
\hline Familia & 53 & 62 & 33 & 28 \\
\hline Pareja & 32 & 13 & 38 & 33 \\
\hline Con un amigo & 2 & 2 & - & - \\
\hline \multicolumn{5}{|l|}{ Nivel educativo alcanzado } \\
\hline Primario & 1 & 3 & 12 & 48 \\
\hline Secundario & 57 & 44 & 63 & 38 \\
\hline Superior & 42 & 53 & 25 & 14 \\
\hline \multicolumn{5}{|l|}{ Ocupación } \\
\hline Trabaja fuera del hogar & 65 & 94 & 43 & 6 \\
\hline Ama de casa & 2 & 6 & 8 & 2 \\
\hline Jubilado/a & - & - & 49 & 92 \\
\hline Estudiante & 30 & - & - & - \\
\hline Desocupado & 3 & - & - & - \\
\hline
\end{tabular}


relaciones comunitarias/servicios de salud y relaciones laborales y/o de estudio.

La entrevista estructurada de apoyo social ha mostrado adecuadas propiedades psicométricas a partir de su aplicación en el contexto argentino. Si bien su versión original es en inglés, se utilizó una traducción al español que ha sido aplicada, con anterioridad, de manera satisfactoria en diversas investigaciones en Argentina (Arias, 2004; Arias \& Polizzi, 2013; Polizzi \& Arias, 2014). Dicho instrumento constituye una adecuada operacionalización de la red de apoyo social ya que incorpora la valoración de todas las características incluidas en su definición conceptual. Además, las evaluaciones de la red llevadas a cabo con dicho instrumento han evidenciado correlaciones con medidas de percepción de apoyo y con indicadores de satisfacción tanto en su versión original (Vaux \& Harrison, 1985), como en su traducción al español (Arias, 2004). Estos hallazgos son coincidentes con la teoría y aportan evidencia adicional a las bondades del mencionado instrumento.

\section{Procedimiento}

Para la recolección de la muestra, se asistió a distintos lugares con la intención de obtener una muestra heterogénea. Se eligieron espacios públicos y privados que se encontraban en zonas geográficas diversas y reunían a personas por variados motivos. Para tal fin se acudió a entidades educativas, centros de jubilados, locales comerciales, hogares particulares, organizaciones laborales, talleres de adultos mayores, centros de día, paseos y espacios públicos. En algunos casos se utilizó el método de bola de nieve, que consiste en pedirle a quienes fueron elegidos para participar que propusieran y facilitarán el contacto con personas que, de acuerdo con los criterios de inclusión anteriormente explicitados, pudieran participar en la investigación. De esa manera fue posible ampliar la muestra favoreciendo la inclusión de personas que residieran en hogares particulares y que no participan activamente en organizaciones, que de otro modo no hubiera sido sencillo lograr localizar para su participación.

En el caso de las instituciones se realizó un contacto previo con los directores o encargados de las mismas con la finalidad de obtener los permisos pertinentes. Las personas fueron invitadas a participar voluntariamente. En primer lugar, se procedió a la comunicación y firma del consentimiento informado. En segundo lugar, se completó el cuestionario de datos sociodemográficos $\mathrm{y}$, en último lugar, se realizó la entrevista estructurada de fuentes de apoyo social. La administración de las técnicas descriptas anteriormente se llevó a cabo en el espacio de entrevistas individuales con una duración aproximada de 30 minutos. Los investigadores realizaron la administración de los instrumentos y efectuaron el registro de las respuestas por escrito.

Excepcionalmente se encontraron personas que presentaban deterioro cognitivo aparente y que por tal motivo fueron excluidas de la muestra. Esta evaluación fue realizada por los investigadores a partir de la información obtenida durante el primer tramo de la entrevista. Cabe destacar que los procesos implicados para completar el consentimiento informado, así como para responder al cuestionario de datos sociodemográficos brindan datos al evaluador acerca del estado cognitivo, ya que, entre otros aspectos, incluyen la indagación de la orientación espacial y temporal, así como su capacidad de comprender la información que se le brinda y de resolver las consignas de manera adecuada. Además, los instrumentos de recolección de datos son administrados por psicólogos especializados en gerontología con amplia experiencia en el trabajo de campo con adultos mayores.

El proyecto fue desarrollado respetando los principios éticos para la investigación con seres humanos estipulados por la Declaración de Helsinki (Asociación Médica Mundial, 2013) y la Ley 11044 de 1990 y el Decreto Reglamentario 3385 de 2008. 


\section{Estrategia de análisis}

Los datos recolectados fueron analizados de manera cuantitativa utilizando el programa $R$, versión 3.4.1. Se aplicaron técnicas de estadística descriptiva e inferencial. Para el logro del objetivo 1 se utilizaron las distribuciones de porcentajes. Para alcanzar los objetivos 2 y 3 se aplicó el análisis de varianza. Previamente y mediante la prueba de Levene, se testeó la homogeneidad de las varianzas de las distribuciones. En los casos en que se hallaron diferencias significativas entre las medias, el análisis se complementó con la prueba de contraste a posteriori de Bonferroni para identificar los pares de medias entre los que se observaban dichas diferencias. La misma, además de permitir la comparación por pares, posibilita controlar la tasa de error global. Complementariamente, en dichos casos se calculó el coeficiente Eta cuadrado $\left(\mathrm{n}^{2}\right)$ para conocer el tamaño del efecto. Se utilizó un nivel $\alpha$ de 0.05 .

\section{Resultados}

Como respuesta al objetivo 1, que apuntaba a identificar los vínculos más incluidos (padre, madre, hijo/a, pareja, hermano,/a, amigo/a, etc.) en la red, en cada grupo de edad, las relaciones de amistad fueron las más incluidas en los tres grupos de menos edad, desde un $99 \%$ en los de 20 a 30 años hasta un $92 \%$ en el grupo de 60 a70 años. En el grupo de menos edad, le siguieron los padres/ madres, hermano/a y pareja como los vínculos más incluidos. En el de 40 a 50, a las amistades le continuaron la pareja en segundo lugar, los padres/ madres en tercer lugar y los hijos/as y hermanos/ as en cuarto lugar con igual porcentaje de inclusión. Entre los de 60 a 70 años, a las amistades le siguieron en porcentaje de incorporación los hijos/ as, la pareja y los hermanos/as. Finalmente, en el grupo de más edad, el tipo de vínculo más incluido fue el de los hijos/as, seguido de las amistades, nietos/as, pareja y hermanos/as.
En los cuatro grupos de edad aparecen entre los vínculos más incluidos las amistades, la pareja y los hermanos/as aunque con diferentes porcentajes (tabla 2). Los padres/madres aparecen entre los principales en los grupos de 20 a 30 y de 40 a 50 , mientras que en los de más edad se incluyen con más frecuencia los hijos/as y en el de 80 a 90 también los nietos/as.

En lo que respecta al objetivo 2, en la tabla 3 se muestran los promedios en el tamaño de la red de apoyo por grupo de edad y cómo varían inversamente en función de la edad. El ANOVA mostró la existencia de diferencias significativas, $F(3,396)=16.38, \mathrm{p}=.000$, entre los grupos. $\mathrm{El}$ $\mathrm{n}^{2}$ correspondiente $(0.11)$ mostró un tamaño del efecto elevado. Al analizar los grupos por pares, el contraste a posteriori de Bonferroni evidenció diferencias significativas entre los dos de menor edad con respecto a los de personas mayores: el de 20 a 30 años con el de 60 a 70 y con el de 80 a 90: (diferencia de medias de 2.0, $\mathrm{p}=.004$ y $3.64, \mathrm{p}=.000$ respectivamente), y el de 40 a 50 años con el de 60 a 70 y con el de 80 a 90 : diferencia de medias de $1.65, \mathrm{p}=.030$ y de $3.2, \mathrm{p}=.000$ respectivamente), así como entre los de 60 a 70 años respecto de los de 80 a $90(1.63 \mathrm{p}=.033)$. En todos los casos el promedio de vínculos resultó menor en el grupo de más edad (tabla 3 ).

Frente al objetivo 3, si consideramos los cuatro tipos de relaciones en las que se distribuyen los vínculos en el mapa de la red: a) amistades, b) familiares, c) relaciones comunitarias/servicios de salud y d) compañeros de trabajo y/o estudio, el ANOVA mostró diferencias significativas en las puntuaciones medias de tres de ellos según el grupo de edad (tablas 4, 6 y 7; figura 1).

En la tabla 4 puede observarse que el promedio de amistades presenta diferencias significativas por grupo de edad: $F(3,396)=23.38, \mathrm{p}=.000 . \mathrm{El} \mathrm{n}^{2}$ de 0.14 evidencia un tamaño del efecto elevado.

El contraste de Bonferroni mostró que los dos grupos de menos edad presentaron promedios más elevados que los obtenidos por los adultos mayores: 
Tabla 2.

Vinculos incluidos en la red de apoyo social por grupo de edad

\begin{tabular}{|c|c|c|c|c|c|c|c|c|c|c|c|c|c|c|c|c|c|c|c|c|c|c|}
\hline & & 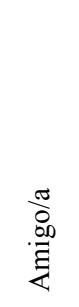 & $\stackrel{\frac{\pi}{O}}{:}$ & $\begin{array}{l}\frac{\pi}{\widetilde{d}} \\
\stackrel{\vec{d}}{a} \\
0\end{array}$ & 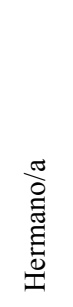 & 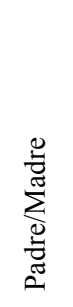 & $\frac{\pi}{\circ}$ & 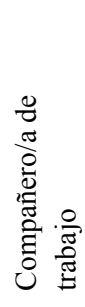 & 兽 & 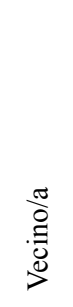 & 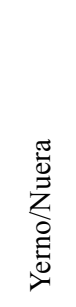 & $\begin{array}{l}\frac{\pi}{O} \\
\stackrel{\Xi}{\Xi} \\
0\end{array}$ & 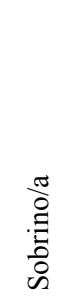 & $\underset{i}{\stackrel{\pi}{\rho}}$ & 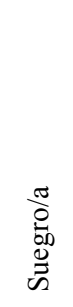 & $\begin{array}{l}\frac{\pi}{0} \\
80 \\
0 \\
00 \\
.0 \\
n \\
0\end{array}$ & 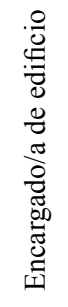 & $\begin{array}{l}\frac{\pi}{0} \\
\frac{0}{0} \\
\frac{0}{4}\end{array}$ & 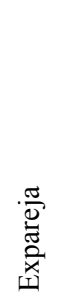 & 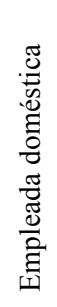 & $\stackrel{\widetilde{G}}{\Xi}$ & 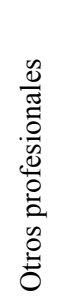 \\
\hline & $n:$ & $\%$ & $\%$ & $\%$ & $\%$ & $\%$ & $\%$ & $\%$ & $\%$ & $\%$ & $\%$ & $\%$ & $\%$ & $\%$ & $\%$ & $\%$ & $\%$ & $\%$ & $\%$ & $\%$ & $\%$ & $\%$ \\
\hline $20-30$ & 100 & 99.0 & 3.0 & 63.0 & 74.0 & 94.0 & 3.0 & 23.0 & 18.0 & 3.0 & 0.0 & 14.0 & 8.0 & 22.0 & 14.0 & 7.0 & 0.0 & 18.0 & 5.0 & 1.0 & 2.0 & 1.0 \\
\hline $40-50$ & 100 & 96.0 & 52.0 & 63.0 & 52.0 & 56.0 & 3.0 & 33.0 & 25.0 & 17.0 & 6.0 & 11.0 & 15.0 & 10.0 & 16.0 & 11.0 & 1.0 & 0.0 & 7.0 & 3.0 & 4.0 & 6.0 \\
\hline $60-70$ & 100 & 92.0 & 80.0 & 49.0 & 38.0 & 17.0 & 20.0 & 14.0 & 18.0 & 23.0 & 26.0 & 16.0 & 12.0 & 4.0 & 1.0 & 4.0 & 3.0 & 0.0 & 3.0 & 3.0 & 3.0 & 0.0 \\
\hline $80-90$ & 100 & 80.0 & 83.0 & 42.0 & 31.0 & 0.0 & 53.0 & 6.0 & 11.0 & 19.0 & 29.0 & 13.0 & 11.0 & 1.0 & 0.0 & 5.0 & 15.0 & 0.0 & 0.0 & 6.0 & 4.0 & 5.0 \\
\hline Total & 400 & 91.8 & 55.0 & 53.0 & 48. & 41.8 & 19.8 & 19.0 & 18. & 56 & 15 & 3 & 15 & 9.3 & 7.8 & 6.8 & 4.8 & 4.5 & 3.8 & 3.3 & 3.3 & 3.0 \\
\hline
\end{tabular}

Tabla 3.

Descriptivos e intervalos de confianza para la media de tamaño de la red de apoyo social según grupo de edad

\begin{tabular}{cccc}
\hline & & \multicolumn{3}{c}{ Vinculos que conforman la red } \\
\hline Grupo de edad & $\mathrm{n}:$ & $\mathrm{M}(\mathrm{DE})$ & $95 \% \mathrm{IC}$ \\
\hline $20-30$ & 100 & $11.99_{\mathrm{a}}(4.47)$ & {$[11.10,12.88]$} \\
\hline $40-50$ & 100 & $11.63_{\mathrm{a}}(4.45)$ & {$[10.75,12.51]$} \\
\hline $60-70$ & 100 & $9.99_{\mathrm{b}}(4.11)$ & {$[9.17,10.79]$} \\
\hline $80-90$ & 100 & $8.35_{\mathrm{c}}(3.41)$ & {$[7.67,9.03]$} \\
\hline Total & 400 & $10.49(4.36)$ & {$[10.06,10.92]$} \\
\hline
\end{tabular}

Notas: las medias que se encuentran en la misma columna y que presentan distintos subíndices, difieren a una $p<.05$ de acuerdo con el procedimiento de Bonferroni; $\mathrm{IC}=$ intervalos de confianza.

el de 20 a 30 años respecto del de 60 a 70 y del de 80 a 90: (diferencia de medias de 2.1, $\mathrm{p}=.000$ y de $3.7, \mathrm{p}=.000$, respectivamente) y el de 40 a 50 años respecto del de 60 a 70 y del de 80 a 90 (diferencia de medias de 1.4, $\mathrm{p}=.022$ y de $2.9, \mathrm{p}=.000$, respectivamente); a su vez, el grupo de adultos mayores jóvenes mostró una media más alta que el de 80 a 90 (diferencia de medias $=1.58, \mathrm{p}=.006$ ).

Como puede observarse en la tabla 5 , no se hallaron diferencias significativas entre los promedios de vínculos familiares incluidos en la red de los cuatro grupos de edad: $F(3,396)=$ $0.33, \mathrm{p}=.8$.

Respecto de las relaciones comunitarias y servicios de salud, se observaron diferencias estadísticamente significativas entre los promedios de los cuatro grupos de edad: $F(3,396)=6.55$, $\mathrm{p}=.000$. El grupo de 20 a 30 años fue el que obtuvo el promedio más bajo mostrando diferencias significativas con los otros tres grupos de edad: con el de 40 a $50(-0.33, \mathrm{p}=.033)$; con el de 60 
a $70(-0.40, \mathrm{p}=.005)$, y con el de 80 a $90(-0.49$, $\mathrm{p}=.000)$ (tabla 6). Para esta variable el tamaño del efecto resultó medio $\left(n^{2}=0.05\right)$

En el caso de compañeros/as de trabajo y/o de estudio se observaron diferencias significativas entre los promedios de los grupos: $F(3,396)=8.26, \mathrm{p}=.000$.
El grupo de 40 a 50 años obtuvo la puntuación media más elevada, evidenciando diferencias significativas respecto de la obtenida por el grupo de 60 a $70(0.53 \mathrm{p}=.004)$, y por el de 80 a $90(0.74$, $\mathrm{p}=.000)$ (tabla 7). Respecto del tamaño del efecto se observó un $\mathrm{n}^{2}=0.06$, indicando un valor medio.

Tabla 4.

Descriptivos e intervalos de confianza para la media de relaciones de amistad según grupo de edad

\begin{tabular}{cccc}
\hline & \multicolumn{3}{c}{ Relaciones de amistad } \\
\hline Grupo de edad & $n:$ & $\mathrm{M}(\mathrm{DE})$ & $95 \%$ IC \\
\hline $20-30$ & 100 & $6.4_{\mathrm{a}}(3.6)$ & {$[5.7,7.1]$} \\
\hline $40-50$ & 100 & $5.7_{\mathrm{a}}(3.7)$ & {$[4.99,6.4]$} \\
\hline $60-70$ & 100 & $4.3_{\mathrm{b}}(3.4)$ & {$[3.7,5.0]$} \\
\hline $80-90$ & 100 & $2.7_{\mathrm{c}}(2.5)$ & {$[2.3,3.2]$} \\
\hline Total & 400 & $4.8(3.6)$ & {$[4.4,5.2]$} \\
\hline
\end{tabular}

Notas: las medias que se encuentran en la misma columna y que presentan distintos subíndices, difieren a una $p<.05$ de acuerdo con el procedimiento de Bonferroni; $\mathrm{IC}=$ intervalos de confianza

Tabla 5.

Descriptivos e intervalos de confianza para la media de vínculos familiares según grupo de edad

\begin{tabular}{cccc}
\hline & & Vínculos familiares & \\
\hline Grupo de edad & $n:$ & $\mathrm{M}(\mathrm{DE})$ & $95 \%$ IC \\
\hline $20-30$ & 100 & $4.8(2.2)$ & {$[4.43,5.32]$} \\
\hline $40-50$ & 100 & $4.5(2.6)$ & {$[4.00,5.05]$} \\
\hline $60-70$ & 100 & $4.7(2.9)$ & {$[4.15,5.30]$} \\
\hline $80-90$ & 100 & $4.8(2.8)$ & {$[4.26,5.35]$} \\
\hline Total & 400 & $4.7(2.6)$ & {$[4.47,4.99]$}
\end{tabular}

Nota: $\mathrm{IC}=$ intervalos de confianza.

Tabla 6.

Descriptivos e intervalos de confianza para la media de relaciones comunitarias y servicios de salud según grupo de edad

\begin{tabular}{cccc}
\hline & \multicolumn{3}{c}{ Relaciones comunitarias/servicios de salud } \\
\hline Grupo de edad & $n:$ & $\mathrm{M}(\mathrm{DE})$ & $95 \%$ IC \\
\hline $20-30$ & 100 & $0.16_{\mathrm{a}}(0.44)$ & {$[0.07,0.24]$} \\
\hline $40-50$ & 100 & $0.49_{\mathrm{b}}(0.90)$ & {$[0.31,0.67]$} \\
\hline $60-70$ & 100 & $0.53_{\mathrm{b}}(0.95)$ & {$[0.37,0.75]$} \\
\hline $80-90$ & 100 & $0.65_{\mathrm{b}}(0.92)$ & {$[0.47,0.83]$} \\
\hline Total & 400 & $0.46(0.85)$ & {$[0.38,0.55]$}
\end{tabular}

Notas: las medias que se encuentran en la misma columna y que presentan distintos subíndices, difieren a una $p<.05$ de acuerdo con el procedimiento de Bonferroni; $\mathrm{IC}=$ intervalos de confianza. 
Tabla 7.

Descriptivos e intervalos de confianza para la media de compañeros de trabajo/estudio según grupo de edad

\begin{tabular}{cccc}
\hline & \multicolumn{3}{c}{ Compañeros/as de trabajo/estudio } \\
\hline Grupo de edad & $n:$ & $\mathrm{M}(\mathrm{DE})$ & $95 \%$ IC \\
\hline $20-30$ & 100 & $0.50_{\mathrm{ab}}(1.1)$ & {$[0.27,0.73]$} \\
\hline $40-50$ & 100 & $0.87^{\mathrm{a}}(1.5)$ & {$[0.57,1.17]$} \\
\hline $60-70$ & 100 & $0.33_{\mathrm{b}}(0.84)$ & {$[0.17,0.51]$} \\
\hline $80-90$ & 100 & $0.13_{\mathrm{b}}(0.58)$ & {$[0.01,0.24]$} \\
\hline Total & 400 & $0.46(1.1)$ & {$[0.35,0.57]$}
\end{tabular}

Notas: las medias que se encuentran en la misma columna y que presentan distintos subíndices, difieren a una $p<.05$ de acuerdo con el procedimiento de Bonferroni; $\mathrm{IC}=$ intervalos de confianza.

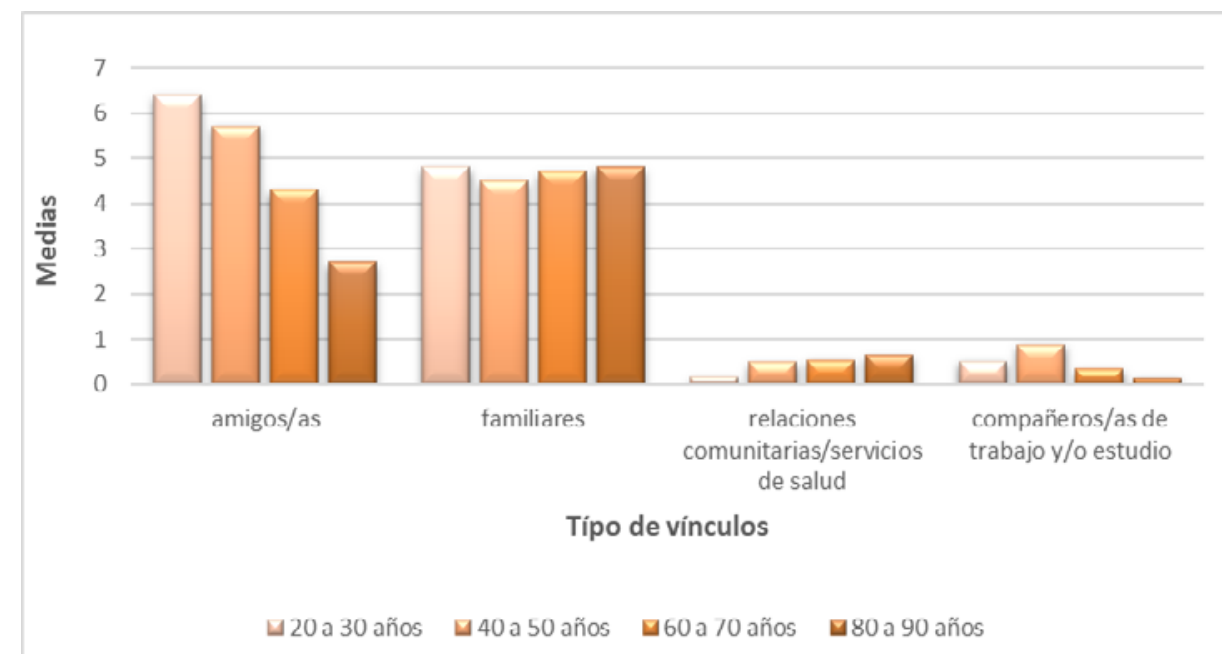

Figura 1. Puntuaciones medias de tipos de vínculos que conforman la red de apoyo social según grupo de edad

\section{Conclusiones}

Los resultados alcanzados permiten observar variadas diferencias en lo que respecta a las redes de apoyo de las personas de distintos grupos de edad. Si bien se trata de un estudio retrospectivo con limitaciones para verificar las modificaciones a través del tiempo, dichas diferencias permiten pensar que a lo largo del curso vital se producen cambios en la red tanto cuantitativos como cualitativos. Cuantitativamente, si bien la red familiar se mantiene estable en las distintas etapas vitales, puede notarse que la red global decrece, al igual que la red de amigos, cuya ma- yor disponibilidad de apoyos la tienen los más jóvenes y la menor, los de edad más avanzada. Sin embargo, a pesar de este achicamiento, las redes de las personas de más edad muestran un adecuado tamaño. Además, la gran mayoría de las redes de las personas mayores evidenciaron una composición variada que incluía tanto relaciones familiares, como no familiares.

Estos resultados son consistentes con la teoría de la selectividad socioemocional (Carstensen, 2006), que sostiene que a pesar de que la red disminuya en tamaño, las personas mayores disponen de recursos de apoyo suficientes, ya que los vínculos se optimizan, profundizan y enriquecen. 
Esto último pudo observarse al analizar la composición de las redes que varía cualitativamente en cada etapa vital. De hecho, la existencia de diferencias significativas entre los promedios de tres de los cuatro tipo de vínculos (relaciones laborales/ estudio, comunitarias/servicios de salud y amistades), según el grupo de edad, pueden entenderse a la luz de la etapa vital que se está transitando y de acuerdo con los roles que se desempeñan de manera predominante en cada una de ellas. De este modo, en consonancia con la teoría del convoy, entre los jóvenes y los de mediana edad, los amigos/as se encuentran de manera mayoritaria en relación a los otros dos grupos, mostrando la importancia de este tipo de vínculos en estas etapas de la vida. De hecho, los jóvenes pasan gran parte de su tiempo con amigos, ya sea compartiendo con ellos espacios educativos o el tiempo libre.

Las relaciones laborales/de estudio son más numerosas en la mediana edad, lo que es consistente con la importancia que el trabajo cobra en dicha etapa de la vida en la que las personas suelen lograr su mayor desarrollo laboral y en la que gran parte de sus vidas se organizan en torno a este. Finalmente, las correspondientes a servicios comunitarios y de salud se presentan más frecuentemente en los tres grupos de más edad, en comparación con los jóvenes. Este predominio de servicios comunitarios y de salud se presenta en mayor medida en los grupos de personas mayores, ya que probablemente parte de ellos transitan situaciones de dependencia, requieren ayudas que provienen de estos espacios o de cuidados de parte de técnicos o profesionales de la salud para desarrollar su vida cotidiana o mejorar su calidad de vida.

La inclusión o no de ciertas relaciones específicas o su incorporación en diferentes porcentajes en cada etapa vital (eg., padre/madre, hijo/a, pareja, nieto/a etc.) permiten pensar que muchas de ellas se incluyen por nacimiento o se pierden por fallecimiento. Es así que estas diferencias en el porcentaje de inclusión según la etapa vital se presentan de manera más clara en las relaciones con personas que pertenecen a otras generaciones - ya sean anteriores o posteriores-.

Estas variaciones en la composición de las redes según el grupo de edad también pueden entenderse desde la teoría del convoy (Kahn \& Antonucci, 1980), ya que se producirían en la medida en que la persona va transitando distintos eventos vitales. Adicionalmente, esta presencia o no de ciertas relaciones en cada etapa vital muestra la coexistencia de pérdidas y de ganancias de vínculos a lo largo del curso vital.

Asimismo, se observa que algunos vínculos cobran más relevancia en la vejez en comparación con otras etapas de la vida, por ejemplo, los hijos/ as, en forma más marcada, y los nietos/as, lo que posibilita pensar que estas relaciones se optimicen y se vuelvan más centrales y fundamentales cuando se ha perdido a la pareja.

Lo expuesto permite pensar en el dinamismo de la red de apoyo que se caracteriza no solo por la pérdida o selección de algunos vínculos, sino también por el cuidado y profundización de los que se poseen (optimización) y la incorporación de nuevas relaciones (compensación). Esto evidencia un proceso de adaptación como el propuesto por Baltes y Freund (2007).

Sin embargo, puede suponerse que este dinamismo en la red implica un impacto a nivel subjetivo. Cabe destacar que al tratarse de vínculos que forman parte de la red de apoyo, las modificaciones que en ellos se producen tienen, en la mayoría de los casos, un importante efecto - ya sea positivo o negativo - sobre el bienestar de la persona. Por ello, es importante continuar profundizando el conocimiento acerca de las características y de los cambios de los apoyos sociales de las personas de edad avanzada y estudiar los efectos que ellos tienen a nivel subjetivo. Esto resulta significativo para conocer los recursos de los que disponen para hacer frente a situaciones de cambio y pérdidas, así como para desarrollar dispositivos de intervención que permitan fortalecer los apoyos sociales. Al respecto, sería relevante avanzar en la exploración 
de las redes de los mayores de 90 años, así como en la generación de propuestas que favorezcan el contacto social y la posibilidad de generar nuevos vínculos. Dicho grupo poblacional, cada vez más numeroso, nos enfrenta a involucrarnos en problemáticas emergentes, en muchos casos inéditas, así como a generar nuevas estrategias que brinden respuestas a estas.

\section{Referencias}

Acrich, L. (2012). La psicogerontología social y la evaluación de las redes sociales de apoyo. ECOS, 2(1), 17-31. http://www.uff.br/periodicoshumanas/index.php/ecos/article/view/842

Antonucci, T. C. (2001). Social relations. An examination of social networks, social support, and sense of control. En J. E. Birren \& K. W. Schaie (Eds.), Handbook of the psychology of aging (pp. 427-453). Academic Press.

Antonucci, T., Arjrouch, K., \& Birditt, K. (2014). The Convoy model: Explaining social relations from a multidisciplinary perspective. The Gerontologist, 54(1), 82-92.

Arias, C. (2004). Red de apoyo social y bienestar psicológico en personas de edad. Suárez.

Arias, C. (2009). La red de apoyo social. Evaluación e intervención con personas adultas mayores. Revista de Psicologia da IMED. 1(1),147-158.

Arias, C. (2013). El apoyo social en la vejez: la familia, los amigos y la comunidad. Revista Kairós Gerontologia. 16(6), 25-40.

Arias, C. (2015a). Redes sociales de las personas mayores. En M. Roque \& A. Fassio (Comp.), Políticas públicas sobre envejecimiento en los países del Cono Sur (pp. 267-291). Flacso/BID.

Arias, C. (2015b). La red de apoyo social. Cambios a lo largo del ciclo vital. Revista Kairós Gerontología. 18(20),149-172.

Arias, C. (2019). Características de la red de apoyo social y cambios por pérdida e incorporación de vínculos en adultos de mediana edad y adultos mayores (Tesis de Doctorado, Universidad Nacional de Mar del Plata, Mar del Plata).

Arias, C., \& Polizzi, L. (2013). The couple relationship. Support functions and sexuality in old age. Revista Kairós Gerontologia, 16(1), 27-48.

Asociación Médica Mundial- AMM. Declaración de Helsinski. Principios éticos para las investigaciones con seres humanos. Sesagesimacuarta Asamblea General, Fotaleza, Brasil, Octubre de 2013.

CEPAL (2004). Estrategia regional e implementación para América Latina y el Caribe del Plan de acción internacional de Madrid sobre el envejecimiento. Autor.

Baltes, P. B., \& Freund, A. M. (2007). El potencial humano como orquestación de la sabiduría y la optimización selectiva por compensaciones. En L. G. Aspinwall \& U. M. Staudinger (Eds.), Psicología del potencial humano. Cuestiones fundamentales y normas para una psicologia positiva (pp. 45-61). Gedisa.

Becker C., Kirchmaier I., \& Trautmann, S. T. (2019). Marriage, parenthood and social network: Subjective well-being and mental health in old age. PLoS ONE, 14(7). https://doi.org/10.1371/ journal.pone. 0218704

Bozo, O., Toksabay, N., \& Kürüm, O. (2009). Activities of daily living, depression, and social support among elderly Turkish people. Journal of Psychology, 143, 193-205.

Carstensen, L. L. (2006). The influence of a sense of time on human development. Science, 312, 1913-1915.

Carstensen, L., \& Charles, S.T. (2007) El envejecimiento humano: ¿por qué incluso las buenas noticias se toman como malas? En Aspinwall, L. G. \& Staudinger, U. M. (Eds.), Psicología del potencial humano. Cuestiones fundamentales y normas para una psicología positiva (pp. 111- 126). Gedisa.

Cohen S. (2020). Psychosocial vulnerabilities to upper respiratory infectious illness: Implications for susceptibility to coronavirus disease 
2019 (COVID-19). Perspectives on psychological science: A journal of the Association for Psychological Science, 1745691620942516. https://doi.org/10.1177/1745691620942516

Conway, F., Magai, C., Jones, S., Fiori, K., \& Gillespie, M. (2013). International Journal of Aging and Human Development, 76(1), 01-27.

Cornwell, B., \& Laumann, E. O. (2013). The health benefits of network growth: New evidence from a national survey of older adults. Social Science \& Medicine, 1-13.

Cornwell, B., Schumm, L. P., Laumann, E. O., \& Graber, J. (2009). Social networks in the NSHAP study: Rationale, measurement, and preliminary findings. Journal of Gerontology: Social Sciences, 64B(S1) i5-i11.

Dabas, E. (1993). Red de redes. Las prácticas de la intervención en redes sociales. Paidós.

Fernández Ballesteros, R., Reig Ferrer, A., \& Zamarrón Casinello, M.D. (2009). Evaluación en psicogerontología. En R. Fernández Ballesteros (Ed.), Psicología de la vejez. Una psicogerontología aplicada (pp.35-96). Pirámide.

Fiori K. L., Antonucci T. C., \& Cortina K. S. (2006). Social network typologies and mental health among older adults. The Journals of Gerontology, Series B: Psychological Sciences and Social Sciences, 61, P25-P32.

Frey, C., \& Roethlisberger, C. (1996). Social support in healthy adolescents. Journal of Youth and Adolescence, 25(1), 17-31.

Gallagher, M., Prinstein, M. J., Simon, V., \& Spirito, A. (2014). Social anxiety symptoms and suicidal ideation in a clinical sample of early adolescents: Examining loneliness and social support as longitudinal mediators. Journal of Abnormal Child Psychology, 42(6), 871-883.

Giles, L., Glonek, G., Luszcz, M., \& Andrews, G. (2005). Effect of social networks on 10-year survival in very old Australians: The Australian longitudinal study of aging. Journal of Epidemiology and Community Health, 59 (7), 574-579.
Gracia Fuster, E. (1997). El apoyo social en la intervención comunitaria. Paidós.

Gyasi, R., Phillips, D., \& Abass, K. (2019). Social support networks and psychological wellbeing in community-dwelling older Ghanaian cohorts. International Psychogeriatrics, 31(7), 1047-1057. https://doi.org/10.1017/ S1041610218001539

Huang, X., Western, M., Bian, Y., Li, Y., Côté, R., \& Huang, Y. (2019). Social networks and subjective wellbeing in Australia: New evidence from a national survey. Sociology, 253(2):401-421. https://doi.org/10.1177/0038038518760211

Johnson, K.D., Whitbeck, L., \& Hoyt, R. (2005). Predictors of social network composition among homeless and runaway adolescents. Journal of Adolescence, 28(2) 231-248.

Kahn, R. L., \& Antonucci, T. C. (1980). Convoys over the life course: Attachment, roles, and social support. En P. B. Baltes, O. Brim, (Eds.), Life-span development and behavior (pp. 254283). Academic Press.

Lee, C., Dickson, D. A., Conley, C., \& Holmbeck, G. (2014). A closer look at self-esteem, perceived social support, and coping strategy: A prospective study of depressive symptomatology across the transition to college. Journal of Social and Clinical Psychology, 33(6), 560-585.

Lomnitz, L. (1975). Cómo sobreviven los marginados. Siglo XXI Editores.

Meléndez Moral, J. C., Tomás Miguel, J. M., \& Navarro Pardo, E. (2007). Análisis de las redes sociales en la vejez a través de la entrevista Manheim. Salud Pública de México, 49(6), 408-414.

Naciones Unidas. (2002). Informe de la Segunda Asamblea Mundial sobre Envejecimiento. Autor.

Okamoto, J., Johnson, C. A., Leventhal, A., Milam, J., Pentz, M. A., Schwartz, D., \& Valente, T. W. (2011). Social network status and depression among adolescents: An examination of social network influences and depressive symptoms 
in a Chinese sample. Research in Human Development, 8(1), 67-88.

Orcasita, L. T., Uribe, A. F., Castellanos, L. P., \& Gutiérrez Rodríguez, M. (2012). Apoyo social y conductas sexuales de riesgo en adolescentes del municipio de Lebrija-Santander. Revista de Psicología (PUCP), 30(2), 371-406.

Organización Mundial de la Salud (2002). Envejecimiento activo: un marco político. Revista Española de Geriatría y Gerontología, 37(S2), 74-105.

Padua, J. (1982). Técnicas de investigación aplicadas a las ciencias sociales. Fondo de Cultura Económica.

Pantusa, J., \& Arias, C. (2017). Vínculos de apoyo en la mediana edad y en la vejez. En C. Arias (Comp.), Mediana edad y vejez. Perspectivas actuales desde la psicología (pp.123-146). UAA.

Plath, D. W (1980). Contours of consociation: Lessons from a Japanese narrative. Life-span Development and Behavior, 3, 287-305.

Polizzi, L., \& Arias, C. (2014). Vínculos que brindan más satisfacción en la red de apoyo social de adultos mayores. Pensando Psicología, 10(17), 61-70.

Provincia de Buenos Aires, Departamento de Salud. Decreto Reglamentario No 3385 (30 diciembre 2008). http://www.ms.gba.gov.ar/ssps/investigacion/marco-normativo/Ley-11044/Decreto-3385.pdf
Provincia de Buenos Aires, Senado y Cámara de Diputados. Ley Provincial No 11044 (6 de diciembre de 1990).

Reyes, C., Camacho, M., Eschbach, K., \& Markides, K. (2006). El contexto de la familia y el vecindario en la salud de los ancianos del estudio EPESE hispano. Colombia Médica, 37, 45-49.

Sánchez Vidal, A. (1990). Psicología comunitaria. Bases conceptuales y métodos de intervención. EUB.

Shirom, A., Toker, S., Alkaly, Y., Jacobson, O., \& Balicer, R. (2011). Work-based predictors of mortality: A 20-year follow-up of healthy employees. American Psychological Association, 30(3), 268-275.

Sluzki, C. (1996). La red social: Frontera de la práctica sistémica. Gedisa.

Vaux, A., \& Harrison, D. (1985). Support network characteristics associated with support satisfaction and perceived support. American Journal of Community Psychology, 13(3), 245-268.

Vivaldi, F., \& Barra, E. (2012). Bienestar psicológico, apoyo social percibido y percepción de salud en adultos mayores. Terapia Psicológica, 30(2), 23-29.

Ye, L., \& Zhang, X. (2019). Social network types and health among older adults in rural China: The mediating role of social support. International Journal of Environmental Research and Public Health, 16(410). https://doi.org/10.3390/ ijerph16030410
Recibido: agosto 14, 2019 fiprobado: diciembre 9, 2020 